\title{
ADVANCED TECHNOLOGIES FOR DECONTAMINATION AND CONVERSION OF SCRAP METALS
}

\section{AUTHORS:}

Thomas R. Muth

John Moore

David Olson

Brajendra Mishra

\section{CONTRACTORS:}

Manufacturing Sciences Corporation

804 Kerr Hollow Road

Oak Ridge, Tennessee 37830

\section{CONTRACT NUMBER:}

DE-AC21-93MC30170

\section{CONFERENCE TITLE:}

Opportunity '95 - Environmental Technology Through Small Business

\section{CONFERENCE LOCATION:}

Morgantown, West Virginia

CONFERENCE DATES:

November 16 - 17, 1994

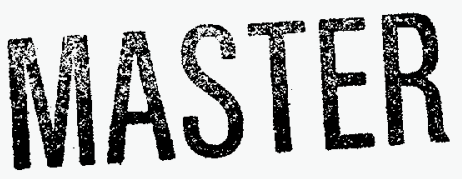

\section{CONFERENCE SPONSOR:}

U.S. Department of Energy - Morgantown Energy Technology Center 


\section{DISCLAIMER}

Portions of this document may be illegible in electronic image products. Images are produced from the best available original document. 

and Conversion of Scrap Metals

CONTRACT INFORMATION

Contract Number

Contractor

Contractor Project Manager

Principal Investigators

METC Project Manager

Period of Performance
DE-AC21-93MC30170

Manufacturing Sciences Corporation

804 Kerr Hollow Road

Oak Ridge, Tennessee 37830

(615) 481-0455

Alan L. Liby

Manufacturing Sciences Corporation

Thomas R. Muth

Colorado School of Mines

Professor John Moore

Professor David Olson

Professor Brajendra Mishra

Jagdish L. Malhotra

September 17, 1993 to November 17, 1994 


\section{INTRODUCTION}

Recycle of radioactive scrap metals (RSM) from decomissioning of DOE uranium enrichment and nuclear weapons manufacturing facilities is mandatory to recapture the value of these metals and avoid the high cost of disposal by burial. The scrap metals conversion project detailed below focuses on the contaminated nickel associated with the gaseous diffusion plants.

Nickel represents 80 percent of the scrap value of all metals that will come from the dismantlemnt of the gaseous diffusion plants. The current DOE nickel inventory is approximately 60,000 tons. 10,000 tons of this material was melted as part of the Cascades upgrade program in the 1970's. There are an additional 50,000 tons in the diffusion plants, much of which is porous and has been in itimate contact with uranium compounds and fission products. Because of the intricacy of the pourous nickel the contamination is not readily removed. Technetium- 99 is known to be particularly troublesome in that regard and is an element that makes a significant contribution to the measured radioactivity.

The melting performed in the 1970 's converted 10,000 tons of diffusion plant nickel into 1 ton cylindrical ingots. No refining of the technetium was accomplished and the cast metal is bulk contaminated with 5 to 10 parts per million by weight (wppm) Technetium. At these contamination levels the nickel activity is 3,000 to 6,000 Bequerels per gram (Bq/gm).

Ultimately recovery of the contaminated nickel will be possible only if the developed reuse process is economically viable and there is a market for the products. The available markets include domestic restricted end use and international unrestricted use.

\section{Restricted End Use}

It is likley that the nickel could find utility in restricted end use products in lieu of domestic unrestricted commerce having no bulk contamination release critera. In that regard the cleanup levels of the bulk material will be less restrictive. As an economic option it is therefore the intent of this study to develop methods to utilize the nickel without decontamination.

Potential restricted end use products may be stainless steel containers for: Vitrification casks; uranium hexafluoride storage; and drums and boxes for waste handling. In addition high nickel alloys represent a product for restricted end use particularly as alloy 825 (a 42\% nickel alloy), a candidate material for the multipurpose cask.

Part of this phase one study incorporated the vacuum induction melting (VIM) of clean stainless steel using commercially available alloys to generate the alloy loss profile peculiar to MSC's steel making process. The alloy loss profile is characteristic to a furnace lining, geometry and melt practice and is necessary information when making steels with precise compositional tolerances.

Using the alloy loss profile data and contaminated nickel as an alloy addition, S30400 stainless steel and inconel 825 were produced.

Ferrochromium, ferromanganese, ferrosilicon, and iron. were added as in conventional steels to produce the alloys. 


\section{Unrestricted End Use}

In contrast to the domestic market for nickel the international market has a criteria for bulk contamination depending on country ranging from 3.7 to 1 Bequerels per gram $(\mathrm{Bq} / \mathrm{gm})$. The international market for the nickel will be available as a reuse option if the nickel can be cleaned to international standards

Several bulk decontamination methods for the 10,000 tons of previously melted nickel have been examined to make use of the nickel in the international marketplace. Dissolution followed by ion exchange and elctrowinning; reactive gas treatment of the liquid metal; and liquid metal treatment with a reactive slag were examined methods.

The dissolution / ion exchange / electrowinning process has shown promise however issues of secondary waste, process control, and process economics have been difficult to overcome. The pyrometallurgical approaches to date have had limited success removing technetium but represent the economic choice for cleanup and reuse when coupled with slag recycling methods

Traditionally high quality slag refined steels are electroslag remelted (ESR) to remove tramp elements. This method effectively removes chosen contaminates from 1000 wppm to 10 wppm and sometimes to the $1 \mathrm{wppm}$ range but does not typically remove contaminants to the parts per billion (ppb) level required for nickel reuse. Inductoslag refining designed in this study was an attempt to improve the cleaning ability of a reactive slag beyond what is achievable by ESR and allow contamination levels to be reduced to the parts per billion level.To achieve this 1000 fold reduction in technetium by slag refining, controlled intimate contact of the reactive slag with liquid nickel was required. This kinetic process depends upon the surface area of the contaminated nickel exposed to the slag for controlled times and temperatures. Design work with that objective was accomplished in phase one of this study.

After system design the work scope was to prove the concept, conduct physical modelling, chemically analyze feed stock, perform refining experiments, do partitioning experiments, evaluate and optimize the refining and then evaluate slag decontamination methods.

\section{EXPERIMENTAL WORK}

\section{Manufacturing of Stainless Steel}

Five 200 pound melts of $S 30400$ stainless steel were melted in MSC's VIM: One using S30400 as feed material; Two using low carbon ferrochromium, ferrosilicon, ferromanganese, flake iron and clean nickel pellets; and two using flake chromium instead of low carbon ferrochromium. The metals were cast from magnesia or alumina crucibles into a graphite mold The chemical analysis of those castings and the accompanying losses are shown in Table 1.

The ingot cast from heat N0061N was rolled according to the rolling schedule shown in Table 2. Tensile bars were removed at various stages during the processing to determine mechanical properties. The mechanical properties of MSC produced stainless steel as well as commercially available material is presented in Table 3 . It can be seen that in the annealed condition the MSC 
produced material is higher in strength and lower in ductility than the commercially available material. It is also too low in ductility to meet the specification of S30400 stainless steel. Metallography was performed on commercially available as well as MSC produced steel and is presented in Figure 1. The MSC produced material shows a slightly work structure, the commercially available material is fully recrystallized. The difference is due to the final heat treatment. The annealing temperature was 950 degrees Celsius and needed increase to 1050 degrees Celsius to accomplish the desired recrystallized microstructure and therefore mechanical properties. At this writing the subsequent heats of materials have not been rolled, annealed, tensile tested and microstructurally characterized, however it is expected that with the 1050 degrees Celsius anneal these materials will meet the specification.

Two melts of S30400 stainless are to be produced from the contaminated nickel using the most favorable loss profile determined from the clean melts above. At this writing those melts have not been produced but are part of the phase one study.

\section{Process Design}

The multipot inductoslag refining unit schematically shown in figure 2 is designed to control the three variables time temperature and surface area exposed to slag. The temperature of the system is controlled by induction heating a graphite susceptor to specific temperatures above the melting point of nickel. The perforated bottom magnesia crucible dictates the surface area of the exposed metal. By controlling orifice size, nickel droplet size and thus surface area are controlled. The slag layer depth and falling velocity of the nickel droplets through the slag layer dictate reaction time. A second inductoslag refining unit is schematically shown in figure 3 . It couples inducto slag refining with the electroslag refining process.

\section{Prove the Concept}

Figure 4 shows the log of the activity of Technetium oxide with the partial pressure of oxygen along with the free energy of formation of the two oxides indicating the potential for removal of technetium by the designed process. Albiet a fission product, Technetium is a group VIIB element on the periodic table and is thought to be chemically similar to Manganese, and Rhenium. An examination of a Richardson Ellingham diagram (figure 5) indicates that technetium (if like manganese) can be removed by an oxidizing slag process without preferentially oxidizing nickel.

The oxidizing slag chosen for this refining work was Calcium Fluoride based. Three fluxes whose composition is shown in Table 4 were chosen with varying oxidation potentials to do the refining.

\section{Surrogate Production}

To establish a nonradioactive Technetium "look a like" a master alloy of nickel 25 wppm Rhenium was cast. This alloy was subjected to the same pyrometallurgical processing as the contaminated nickel with the philosphy that processing of a nonradioactive surrogate could be accomplished in existing research equipment without the 
restrictions of a radiation protection program.

\section{Cold Modelling}

There was utility in cold modelling the nickel / high CaF2 flux system to determine orifice size in the refining experiments as well as establish a method for changing surface area and reaction time in the flux. Liquid Gallium at 50 degrees Celcius was chosen as the nickel substitue and a methanol / antimony chloride mixture as a flux substitute. The properties of the two systems are compared in Table 5. Figure 6 is a plot of Reynolds number versus droplet size comparing the nickel / CaF2 system with the cold model. Similarly figure 7 shows the terminal falling velocity of droplets formed in the cold model compared to the nickel / CaF2 system. A good agreement is recognized.

Once the cold model was established the missing data was the kinetics of the removal reaction. To establish this a chemical analysis of the starting material was required followed by equilibrium partitioning experiments.

\section{Chemical Analysis}

Four ingot pieces of Technetium contaminated nickel provided by the DOE were the starting feed stock for this study. The processing history of the material is speculative but it was thought to be material form one of the 10,000 one ton ingots. It had been melted at least once after the initial ingot casting and perhaps multiple times in experiments that looked at melt refining as a means to decontaminate the ingots. The ingot pieces were "swiss cheese" looking in cross section with pockets of retained flux from previous experiments. They also had the apperance of being drip cast instead of continuously poured.

Sectioning of the ingot pieces followed by quantitative chemical analysis of the machine turnings was performed to establish a baseline Technetium content. The sectioning schematic is shown in figure 8 . The results of the chemical analysis are tabulated in Table 6.

Two quantitive analysis methods were employed: One ignoring the radioactive signiture of the Technetium used direct elemental analysis. The second used radiochemical techniques.

The direct elemental analysis method was by Thermally Activated Resonance Ionization Spectroscopy (TARIS)1. Detection levels in an aqueous solution by the TARIS method are in the range of 0.01 nanograms per milliliter. The second method used contaminated nickel dissolved in an aqeous solution that passed through an ion exchange resin to remove Technetium. The ion exchange resin was then eluted and the eluant evaporated and counted for Technetium. There was no consistent agreement with the two methods.

It is postulated that TARIS sees only the Technetium and that the radiochemical method shows apparent higher Technetium levels due to additional beta contributions from other fission products inherent in the nickel.

Technetium is a low energy beta emitter therefore liquid scintillation is a third method that may be used and at this writing is currently being investigated.

The analytical inconsistances are thought to be caused by inhomogeneous starting material as evidenced by the 
"swiss cheese" cross section and the observance that the ingots look as if they were formed by dripping metal into a pile. Additionally slag entrapment in the metal from previous melts is apparent. A solution to these inhomogenities will be discussed shortly.

Because of the analysis inconsistencies a practical counting method was employed to screen experimental work. Nickel feed stock was premelted in MSC's VIM without flux to form a "button". The "button" was forged into approximately 3 millimeter thick foil and directly counted in a beta counter calibrated for Technetium. Flux reacted nickel was similarly forged and directly counted. Although this technique was not quantitative it represented a practical method to screen results.

\section{Partitioning Experiments}

Partitioning experiments were designed to investiate the removal of trace technetium concentrations from molten nickel by interaction with an oxidizing, high calcium fluoride flux. A schematic of the crucibles used for these experiments is shown in figure 9.

Figures 10, 11, and 12 show the expected results of 11 partitioning experiments. Figure 10 plots technetium concentration versus time holding temperature, flux composition, and surface area to volume ratio constant. Figure 11 holds time and temperature constant and changes the surface area to volume ratio. Figure 12 plots an optimiumized surface to volume ratio against time at constant temperature and changes the flux composition. These plots indicate the expected results however actual results were not as definitive as the plots suggests.

Two preliminary partitioning experiments at the extremes of the anticipated process window were performed to get an indication of the time involved in partitioning experiments. Those extremes were the high oxygen potential flux at high 1650 degrees Celsius for 1 hour and the same flux composition at 1500 degrees Celsius for 2 hours. Using the direct metal counting data for qualitative analysis an $18 \%$ drop in Technetium level was realized in the 2 hours at $\mathbf{1 5 0 0}$ degrees Celsius. The flux evaporated at 1650 degrees Celsius negating results. With the little data available it was determined that Technetium removal to the levels of interest would take 52 hours.

The experimental process window as described in figures 10,11 , and 12 revealed that above 1550 degrees Celsius the flux would evaporate in 6 hours precluding any flux refining beyond that time.

Due to the inconsistencies in the compositional anlysis as described earlier no data is presented. At the time of this writing vacuum induction melting of all the nickel pieces into a single homogeneous ingot is being performed. Additionally the cast ingot will be characterized for Technetium by direct counting, liquid scintillation and by TARIS methods to establish the starting Technetium content.

The reaction times in the partioning experiments required to reduce the technetium in nickel to low levels cannot be reasonably accomplished due to the volitility of the flux. Contaminated nickel pieces from the homogeneous cast ingot will be alloyed with copper to form Monel 400 . This 
lower melting (1320 degree Celsius) alloy will allow the long reaction times to take place without the evaporation of the required slag. Once the kinetics are established the designed inductoslag refining can be performed.

\section{Slag Decontamination}

The decomtamination of slag for reuse back into the inducto slag process is accomplished by either a salt scrub reduction method or an electroscrubbing method. At the present time neither method has been used on Technetium contaminated flux from this study primarily due to the fact that no contaminated flux has been produced, however both processes have been successfully developed and fully tested at the Colorado School of Mines (CSM) for a similar flux -radioactive metal system.

The salt scrubbing reduction method uses a metal reductant $(\mathrm{Ca}, \mathrm{Mg})$ and a gettering metal $(\mathrm{Ga}, \mathrm{Al})$ to reduce the radioactive metal compound and separate the insoluble metal thus produced. The gettering metal helps in achieving high recovery rates of the radionuclide. The reaction is (" $\mathrm{M}$ " stands for the radioactive metal):

$$
4 \mathrm{MO}_{2}+\mathrm{Ca}_{8} \mathrm{Ga} \rightarrow \mathrm{M}_{4} \mathrm{Ga} \downarrow+8 \mathrm{CaO}
$$

The calcium products dissolve in the base flux and are recycled.

The second method electroscrubbing utilizes the electrlytic dissosiation of radioactive compounds over a controlled voltage and current density range.. The voltage is governed by the standard dissociation potential for the compound. The current density dictates the rate of dissociation.

Deposition of the radioactive metal is on a tungsten cathode (stable in fluoride melts) using graphite as the anode. The flux is fully stable during electrolysis due to the very high thermodynamic stability of the constituents such as calcium / aluminum / magnesium /alkali metal; oxides, fluorides or chlorides.

\section{SUMMARY}

Stainless steel can be produced in MSC's VIM to the S30400 specification using nickel as an alloy constituent. Further the cast alloy can be rolled in MSC's rolling mill to the mechanical property specification for S30400 demonstrating the capability to manufacture the contaminated nickel into valuble end products at a facility licensed to handle radioactive materials.

Bulk removal of Technetium from scrap nickel is theoretically possible in a reasonable length of time with the high calcium fluoride flux, however the need for the high temperature creates a practical problem due to flux volatility.

Bulk decontamination is possible and perhaps more desirable if nickel is alloyed with copper to lower the melting point of the alloy allowing the use of the high calcium fluoride flux.

Slag decontamination processes have been suggested which have been proven technically viable at CSM. 
Table 1: Chemical Composition of Cast $\mathbf{S} 30400$ Ingots with Losses

\begin{tabular}{|c|c|c|c|c|c|c|c|}
\hline & C, $\max$ & $\mathrm{Mn}$ & $P$ & $S$ & Si & $\mathrm{Cr}$ & $\mathrm{Ni}$ \\
\hline Spec & $0.08 \max$ & $2.00 \max$ & $0.045 \max$ & $0.03 \max$ & 1.00 max & $18-20$ & $8-10$ \\
\hline & & & & & & & \\
\hline N0061N & 0.067 & 1.35 & NA & $<0.01$ & 0.44 & 18.11 & 8.53 \\
\hline Losses & $\overline{\mathrm{NA}}$ & NA & NA & NA & NA & NA & NA \\
\hline N0088N & $\overline{N A}$ & 0.82 & $\overline{\mathrm{NA}}$ & $\overline{N A}$ & 0.90 & 18.00 & 9.52 \\
\hline Losses & NA & 0.59 & NA & NA & 0.10 & 0.05 & 0.00 \\
\hline N0089N & NA & 0.93 & $\overline{N A}$ & NA & 0.54 & 18.06 & 9.74 \\
\hline Losses & NA & 0.54 & NA & NA & 0.46 & 0.05 & 0.00 \\
\hline N0090N & $\overline{N A}$ & $\overline{N A}$ & NA & $\overline{N A}$ & $\overline{\mathrm{NA}}$ & $\overline{N A}$ & $\overline{N A}$ \\
\hline Losses & $\overline{N A}$ & $\overline{\mathrm{NA}}$ & $\overline{N A}$ & $\overline{N A}$ & $\overline{N A}$ & $\overline{N A}$ & $\overline{\mathrm{NA}}$ \\
\hline N0091N & $\overline{\mathrm{NA}}$ & $\overline{\mathrm{NA}}$ & NA & $\overline{N A}$ & NA & $\overline{\mathrm{NA}}$ & NA \\
\hline
\end{tabular}

Table 2. The Rolling Schedule for Heat N0061N (S30400)

\begin{tabular}{|l|}
\hline Heat @ 1950 deg. F \\
\hline Hot roll from $100 \mathrm{~mm}$ to $12.7 \mathrm{~mm}$ \\
\hline Reheat @ 1950 deg. F \\
\hline Hot roll from $12.7 \mathrm{~mm}$ to $3.3 \mathrm{~mm}$ \\
\hline Shear into 4 pieces $1200 \mathrm{~mm}$ long \\
\hline Reheat pieces 1 and 2 @ 1950 deg. F \\
\hline Hot roll piece 1 to $2.67 \mathrm{~mm}$ thick \\
\hline Hot roll piece 2 to $2.67 \mathrm{~mm}$ thick \\
\hline Reheat piece 2 @ 1950 deg. F \\
\hline Hot roll piece 2 to $1.5 \mathrm{~mm}$ thick \\
\hline
\end{tabular}


Table 3. Mechanical Properties of N0061N (S30400) Compared to Commercially Available S 30400

\begin{tabular}{|l|ccc|}
\cline { 2 - 4 } \multicolumn{1}{c|}{} & $\begin{array}{c}\text { UTS } \\
\text { (ksi) }\end{array}$ & $\begin{array}{c}\text { YLD } \\
\text { (ksi) }\end{array}$ & $\begin{array}{c}\text { \%ELONG } \\
\text { (2 in.GL) }\end{array}$ \\
\cline { 2 - 5 } & & & \\
\hline SPEC: Annealed & 85 & 35 & 50 \\
As Rolled to 3.3 mm & 166.7 & 146.1 & 8.2 \\
Annealed @ 950 deg C & 97 & 45.2 & 50 \\
As Rolled to 2.67 mm & 136.9 & 116.1 & 17.3 \\
Annealed @ 950 deg. C & 102 & 45.6 & 46 \\
Purchased @ 2.67 mm & 96.5 & 43.2 & 58.3 \\
As Rolled @1.5 mm & 136.8 & 118.7 & 13.7 \\
Annealed @ 950 deg C & 102.2 & 52.4 & 37.5 \\
Purchased @ 1.5 mm & 96.5 & 76.7 & 60 \\
\hline
\end{tabular}

Table 4. Flux Compositions Used for Partitioning Experiments

\begin{tabular}{|c|c|c|c|c|}
\hline $\begin{array}{c}\text { Oxygen } \\
\text { Potential }\end{array}$ & $\begin{array}{c}\text { CaF2 } \\
(w+\%)\end{array}$ & $\begin{array}{c}\text { CaO } \\
(w+\%)\end{array}$ & $\begin{array}{c}\text { Al2O3 } \\
(w t \%)\end{array}$ & $\begin{array}{c}\text { Na2O } \\
(w t \%)\end{array}$ \\
\hline High & 85 & 4 & 4 & 7 \\
Medium & 85 & 6 & 4 & 5 \\
Low & 85 & 8 & 4 & 3 \\
\hline
\end{tabular}

Table 5. Cold Model Comparison of Ni / CaF2 with Ga / Methanol-SbCl3

\begin{tabular}{|c|c|c|c|}
\cline { 2 - 4 } \multicolumn{1}{c|}{ Metals } & $\begin{array}{c}\text { Density } \\
\text { (gm/cm3) }\end{array}$ & $\begin{array}{c}\text { Viscosity } \\
\text { (cP) }\end{array}$ & $\begin{array}{c}\text { Surface Tension } \\
\mathrm{mN} / \mathrm{m}\end{array}$ \\
\hline Nickel at 1600 deg. C & 7.74 & 4.12 & 1722.5 \\
\hline Gallium at 50 deg C & 6.08 & 1.93 & $\mathrm{NA}$ \\
\hline Fluxes & & & 288 \\
\hline 90\%CaF2-10\%CaO at 1600 d & 2.44 & 3.2 & NA \\
\cline { 2 - 4 } \\
\cline { 2 - 4 } \\
\hline Methanol-SbCl3 at 50 deg C & 1.82 & 3.2 & $\mathrm{NA}$ \\
\hline
\end{tabular}


Table 6. Technetium Content of DOE Supplied Nickel Pieces

\begin{tabular}{|l|c|c|c|}
\hline Sample \# & $\begin{array}{c}\text { Radio } \\
\text { Chemical } \\
\text { (pCi/gm) }\end{array}$ & $\begin{array}{l}\text { Rad to ppm } \\
\text { Conversion }\end{array}$ & TARIS \\
& & (wppm) \\
\hline $003-12 / 23$ & 3488 & 129 & 0.19 \\
\hline $003-34 / 45$ & 15975 & 592 & 0.26 \\
\hline $003-56 / 67$ & 6036 & 233 & 0.42 \\
\hline $003-78 / 88$ & 37279 & 1381 & 0.27 \\
\hline $004-12 / 23$ & 2377 & 88 & 0.50 \\
\hline $004-34 / 45$ & 3135 & 116 & 0.45 \\
\hline $004-56 / 67$ & 4330 & 160 & 0.24 \\
\hline $004-78 / 88$ & 5985 & 222 & 0.34 \\
\hline $005-12 / 23$ & 15771 & 584 & 0.16 \\
\hline $005-34 / 45$ & 14743 & 546 & 0.26 \\
\hline $005-56 / 67$ & 7527 & 279 & 0.19 \\
\hline $005-78 / 88$ & 19025 & 705 & 0.17 \\
\hline & & & \\
\hline $006-12 / 23$ & 20945 & 776 & 0.31 \\
\hline $006-34 / 45$ & 20701 & 767 & 0.18 \\
\hline $006-56 / 67$ & 35408 & 1311 & 0.30 \\
\hline $006-78 / 88$ & 8207 & 304 & 0.34 \\
\hline
\end{tabular}




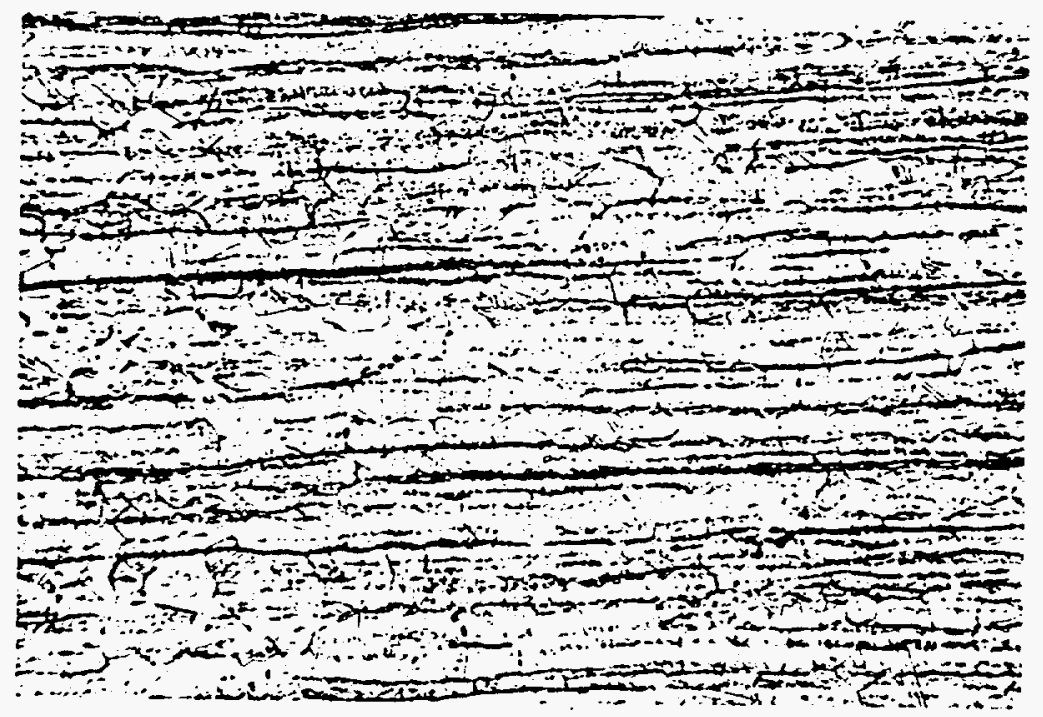

(a)

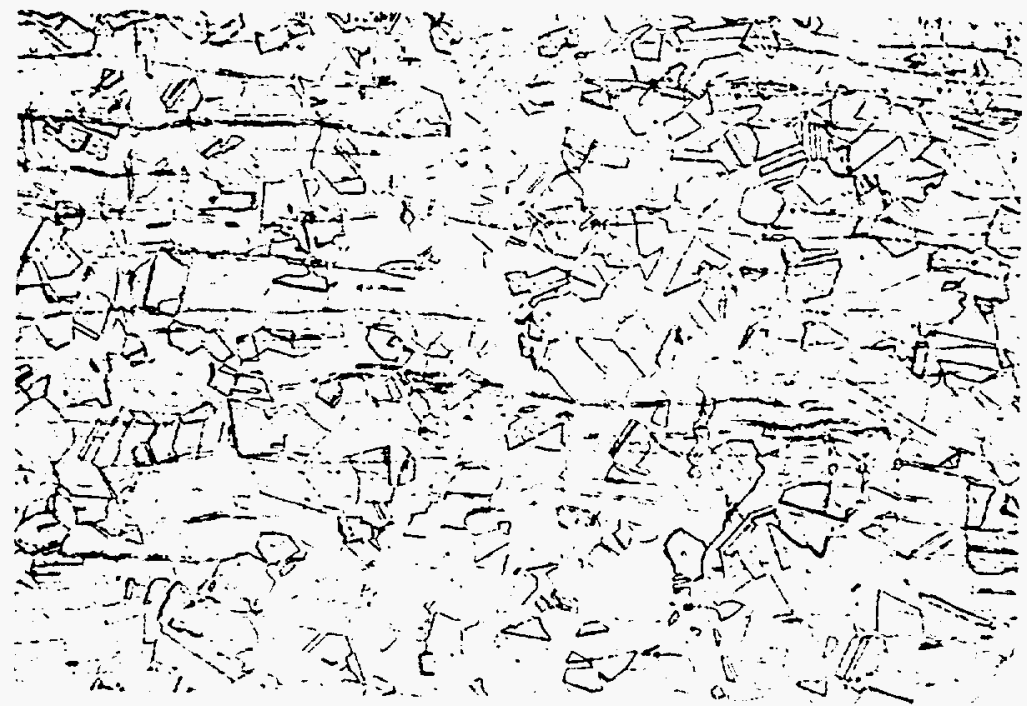

(b)

Figure 1. The Microstructure of MSC Rolled Material (a) and Purchased S30400 (b) $(200 \mathrm{X})$. 


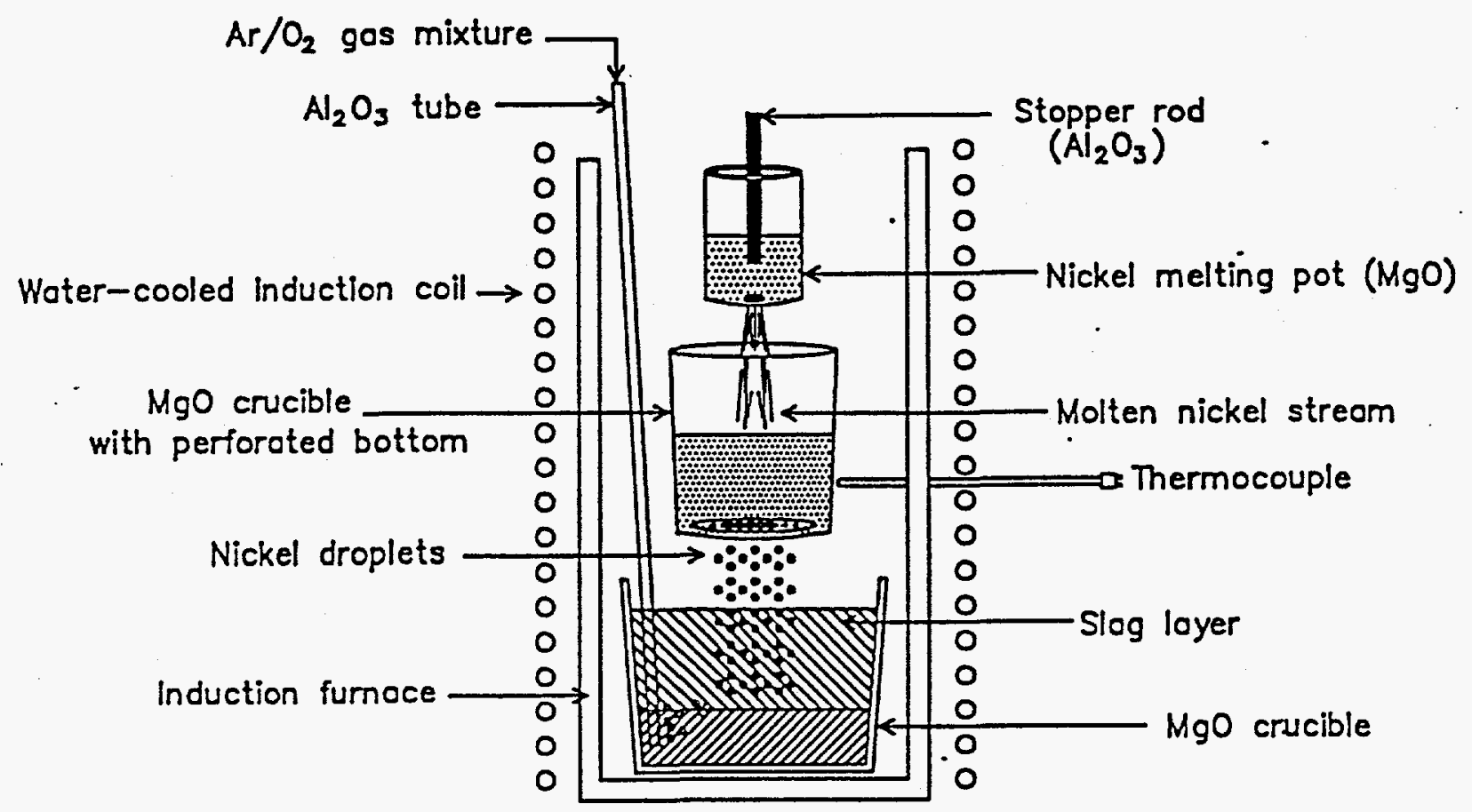

Figure 2. A Batch Type Multi-Pot Inductoslag Refining Unit 


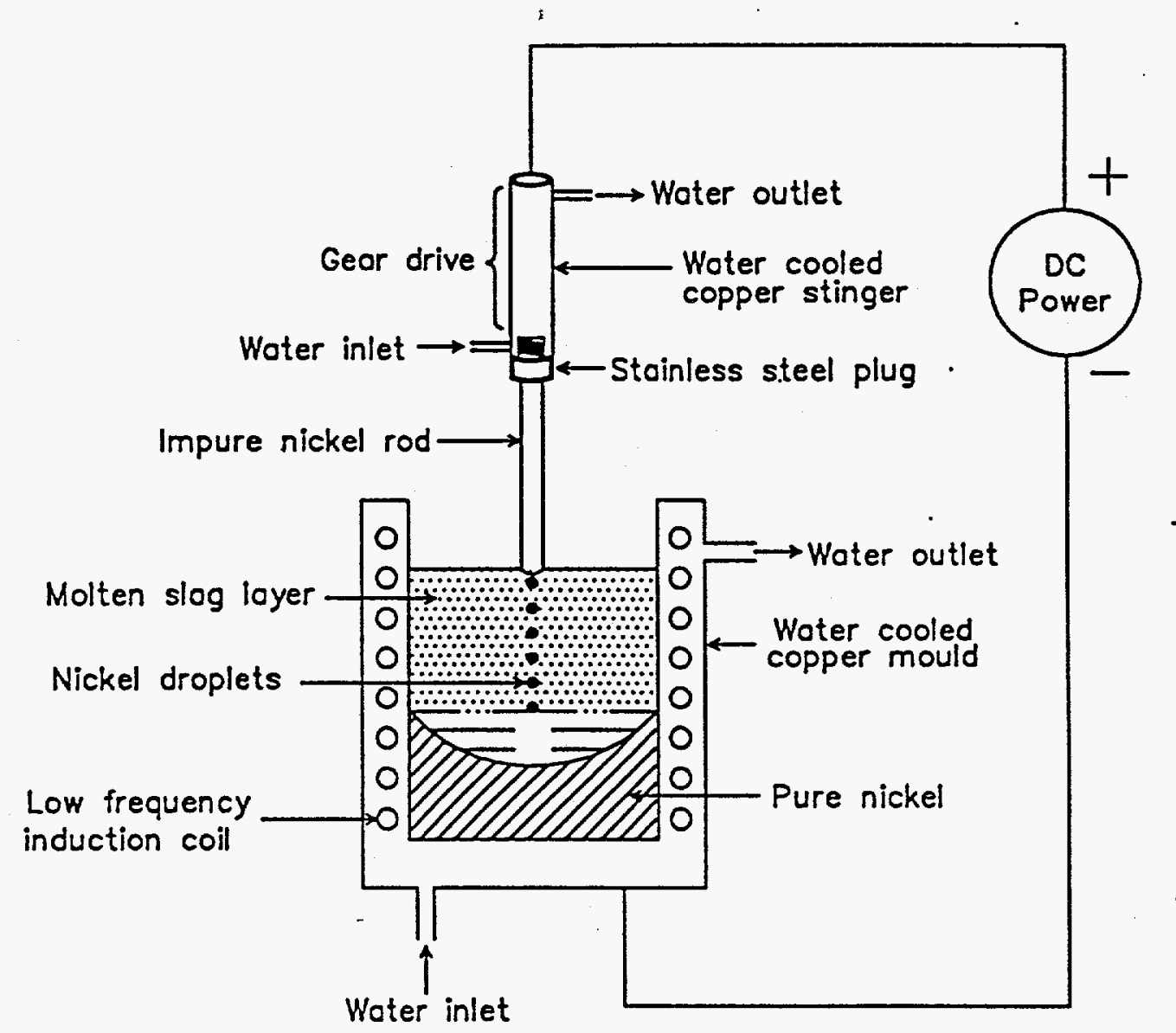

Figure 3. A Batch Type Inductoslag Refining Unit with Electroslag Refining 
TECHNITIUM REMOVAL FROM NICKEL SCRAP INDUCTOSLAG REFINING AT $1700 \mathrm{C}$

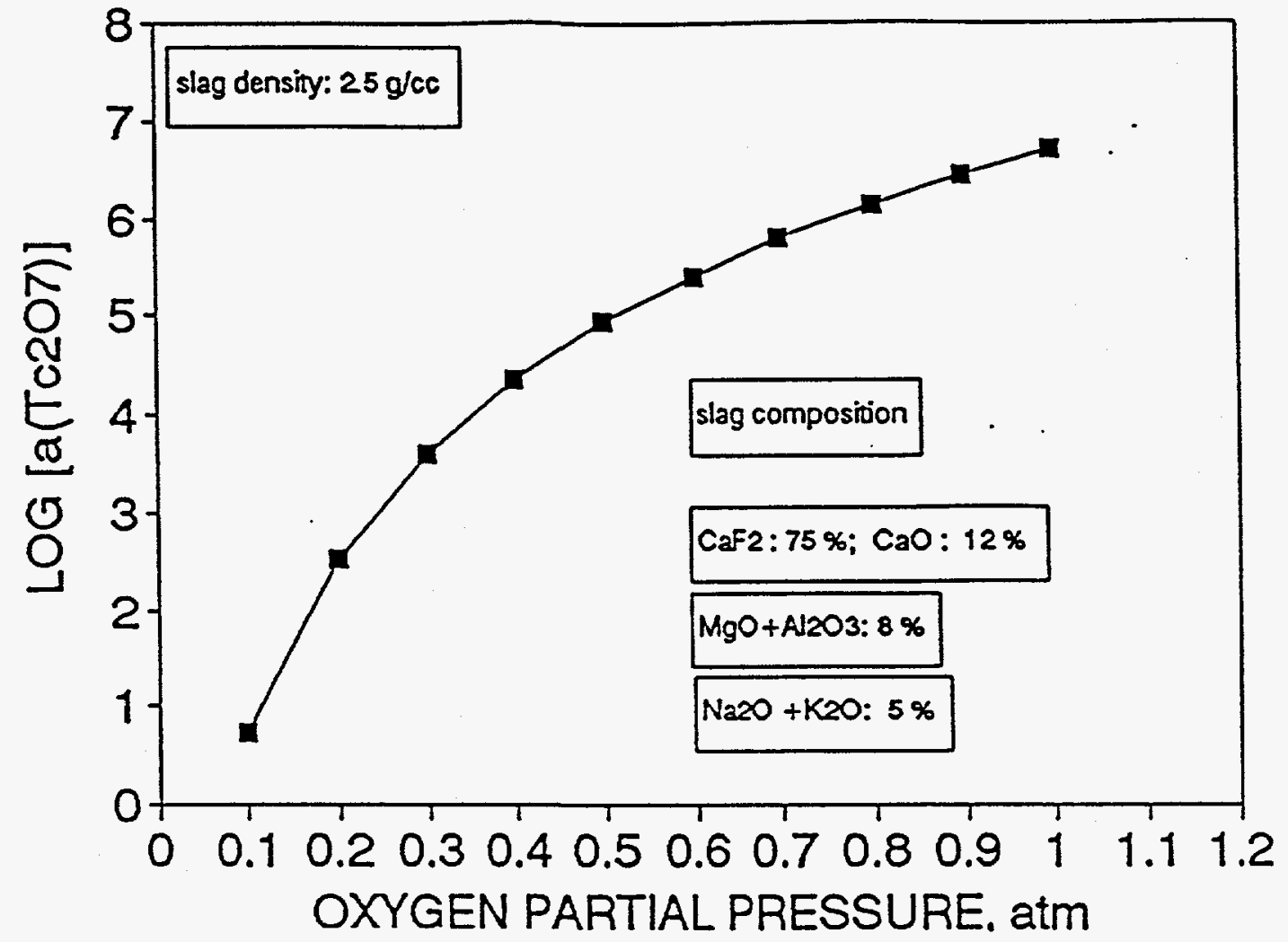

Figure 4. A Plot Depicting the Log of Technetium Oride Activity Versus Oxygen Partial Pressure 


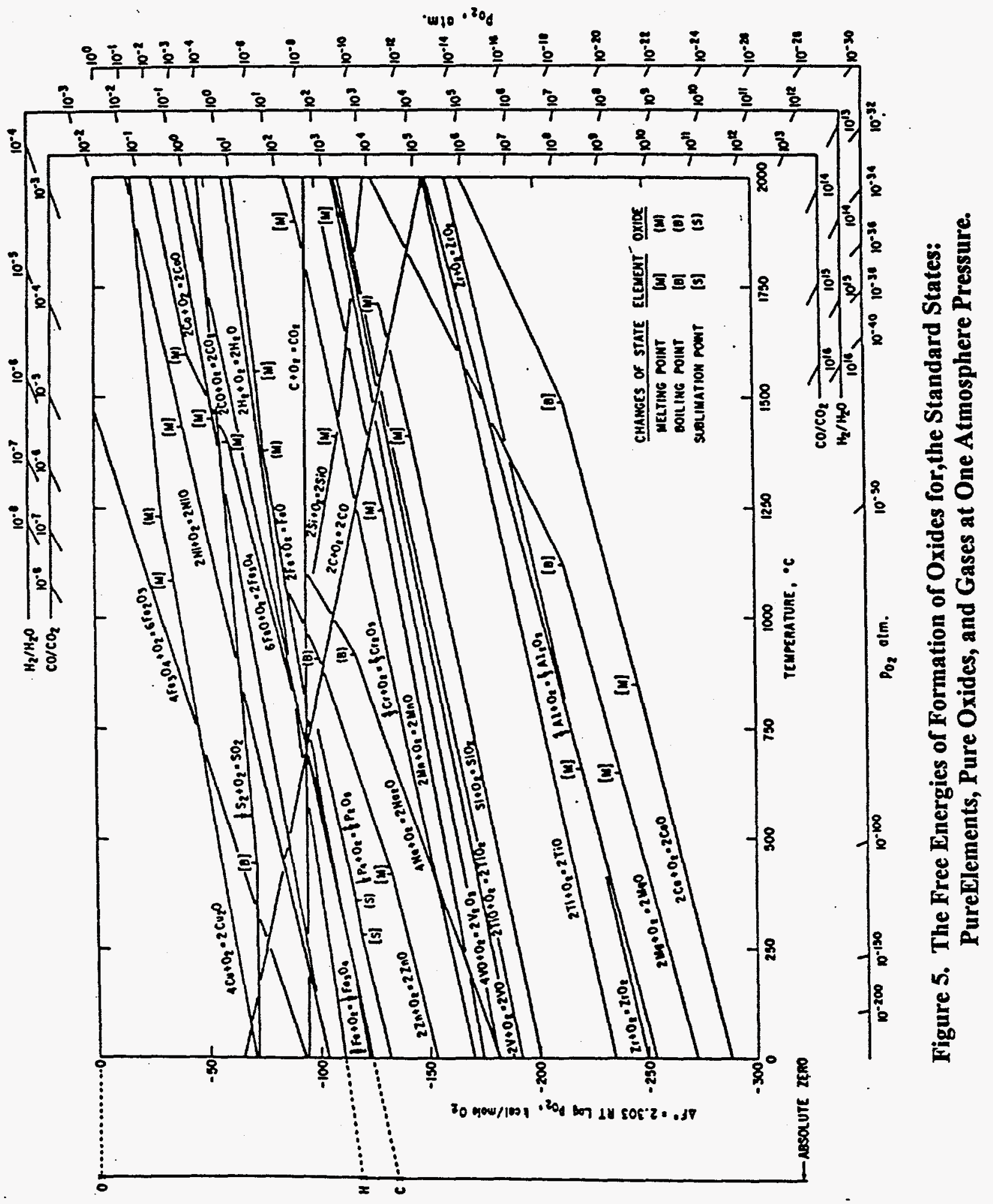




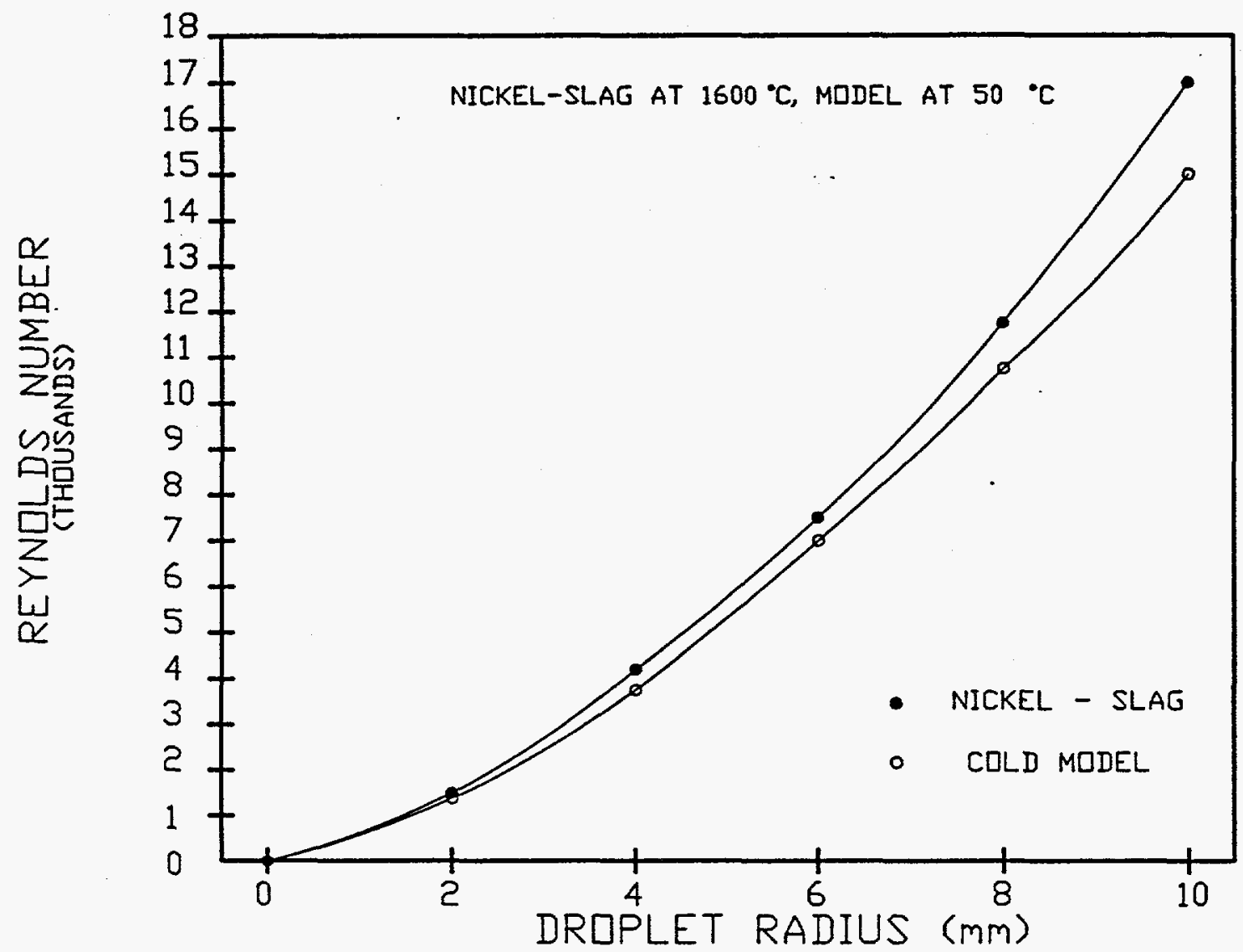

Figure 6. Reynolds Number Versus Droplet Radius for the Nickel / Slag System and the Cold Model System. 


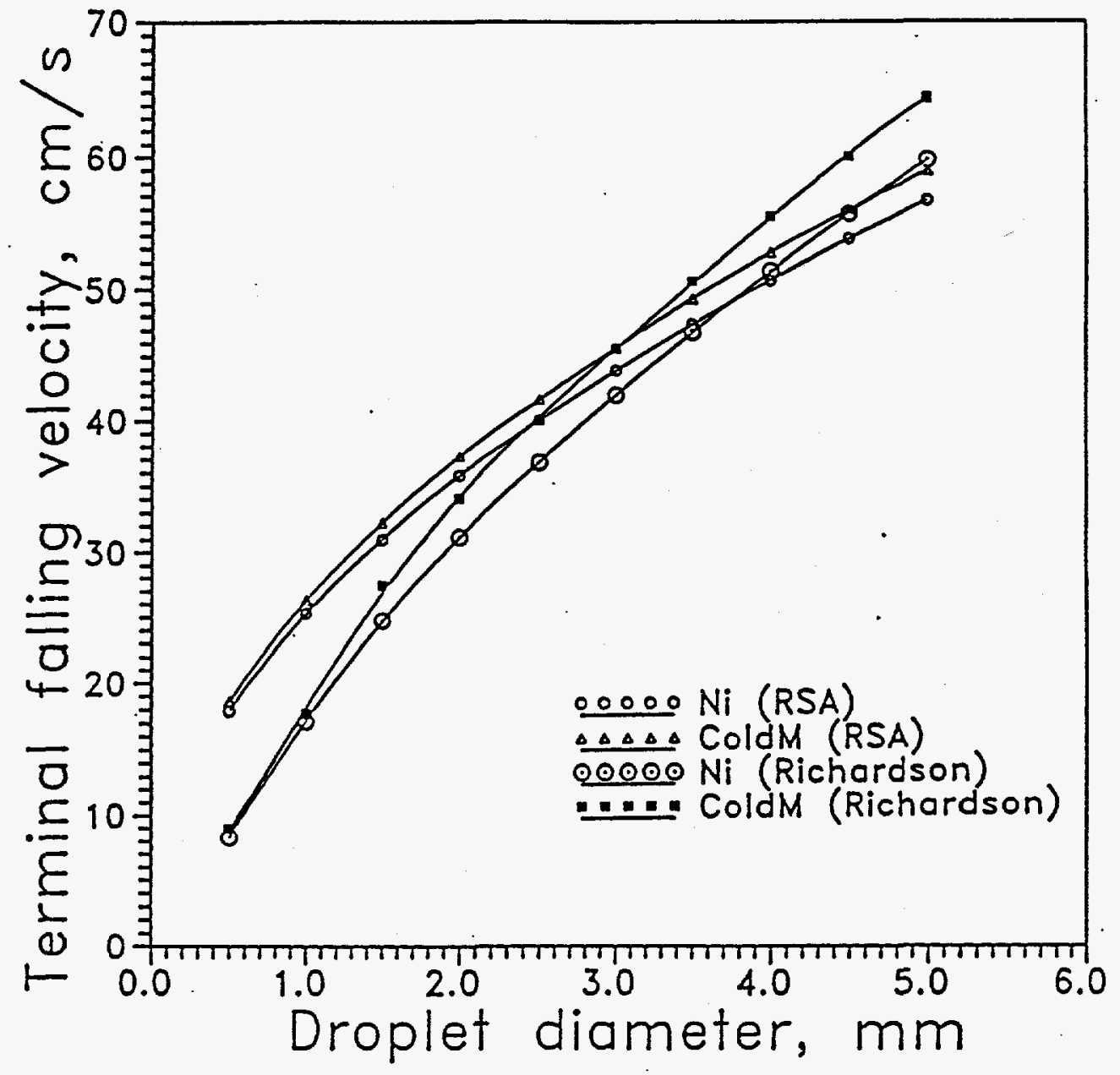

Figure 7. The Terminal Falling Velocity of Droplets Through Slag as a Function of Droplet Diameter. 


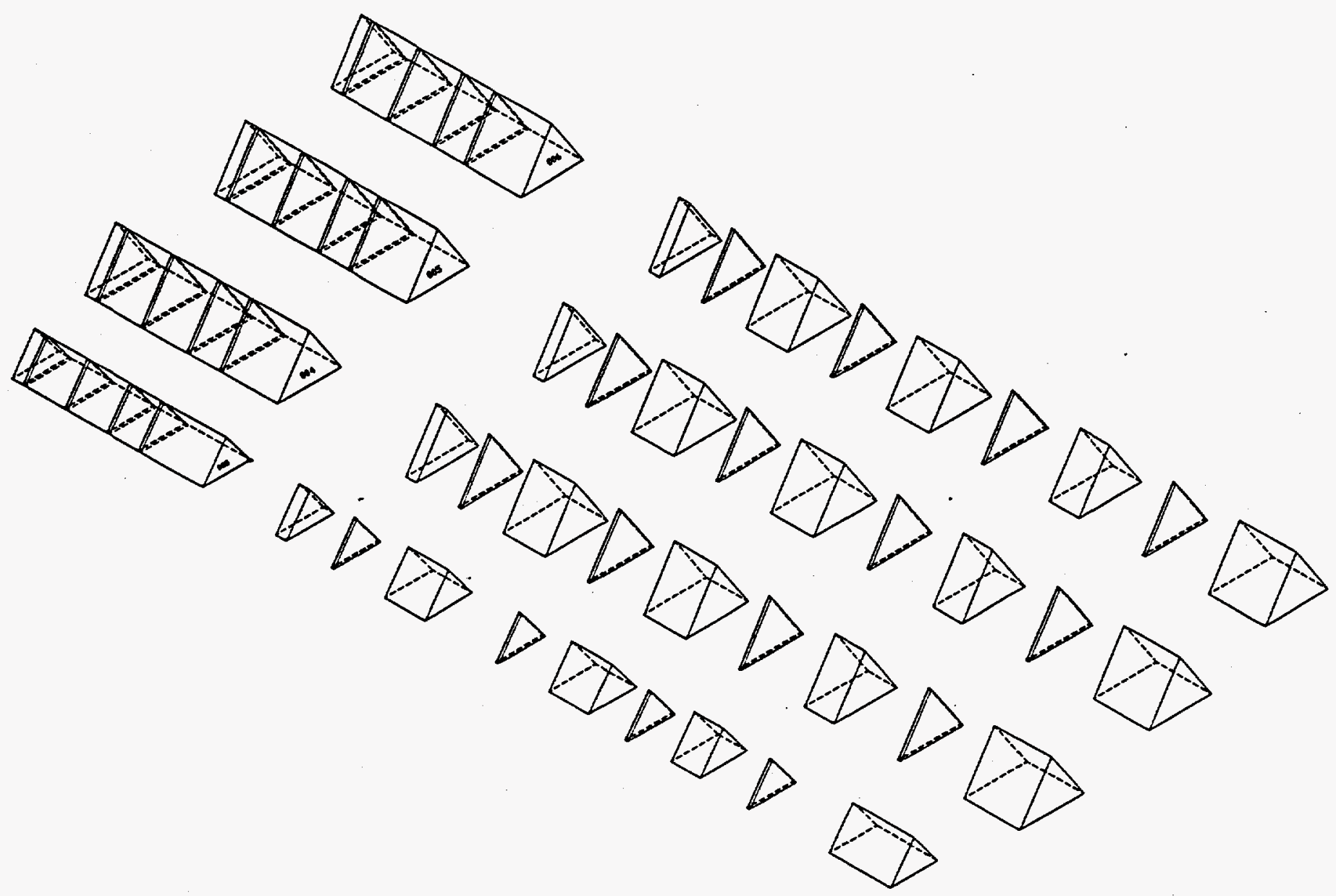

Figure 8. Sectioning and Sampling Schematic Showing the Locations of the Sampling Cuts.

$-224-$ 


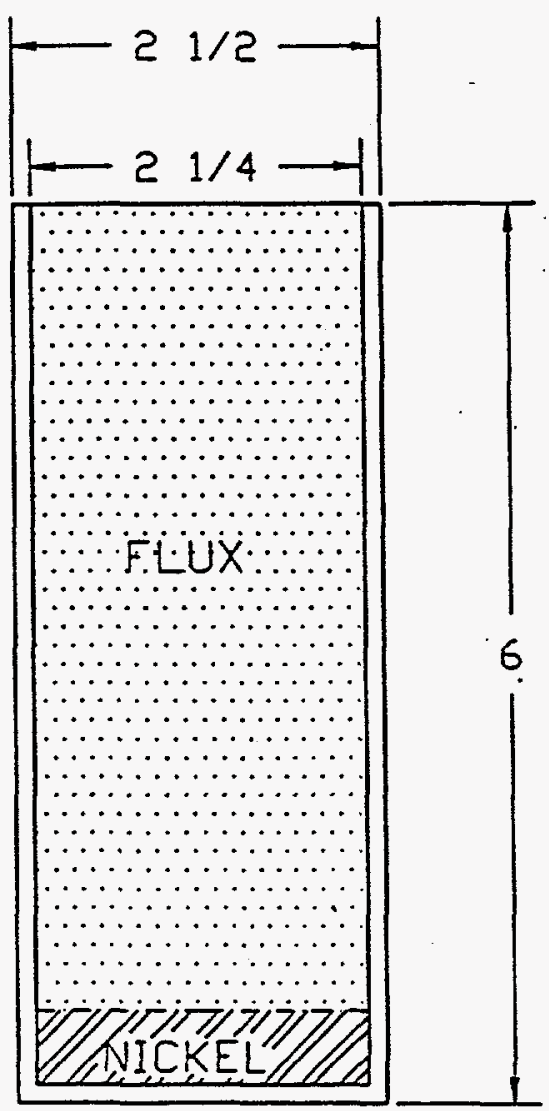

Figure 9. Cross Section of the Magnesia Crucible Used for the Partitioning Experiments 


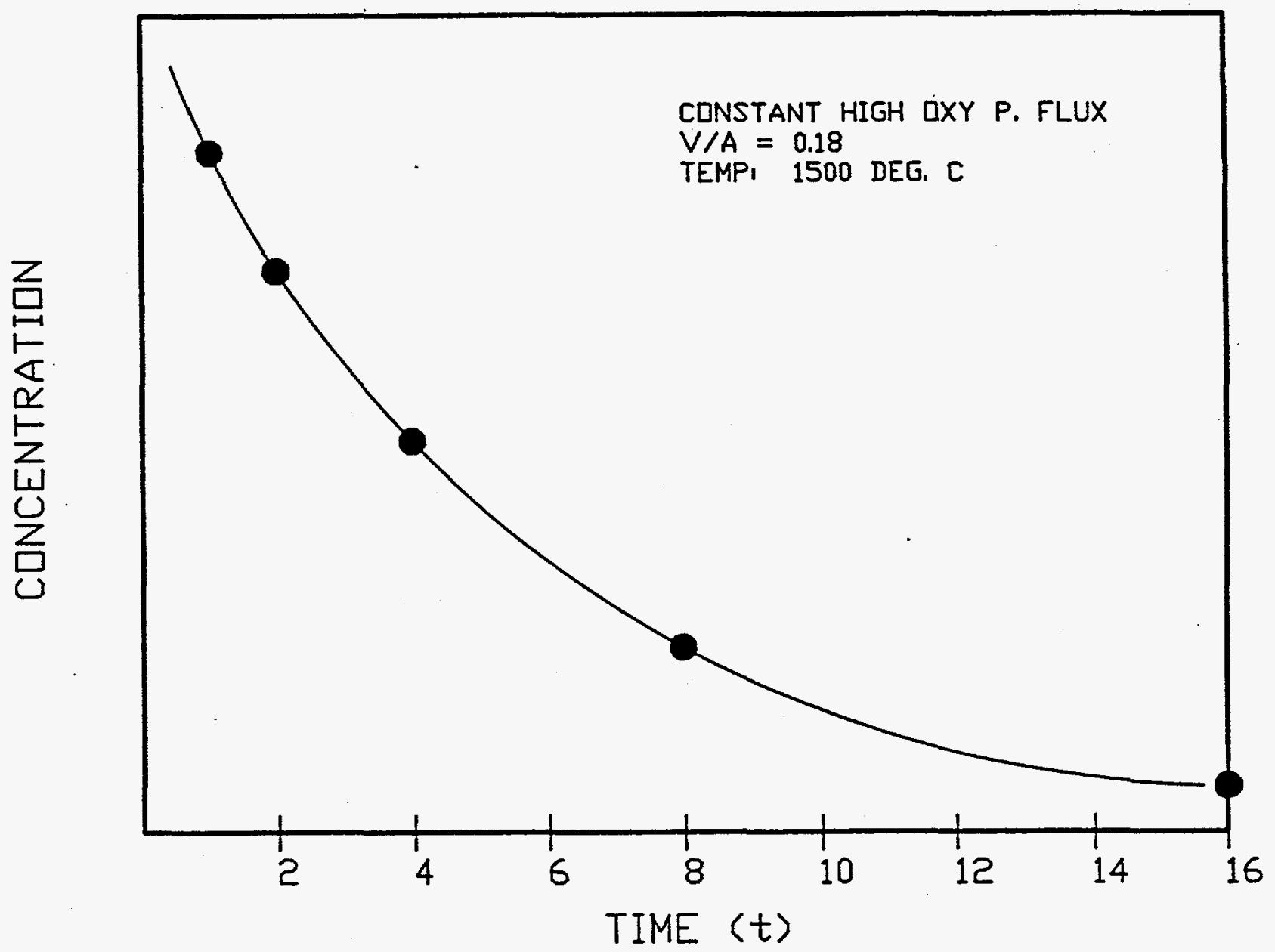

Figure 10. Anticipated Results from the Partitioning Experiments where Volume to Surface Area Ratio of Slag Metal Bath, Temperature, and Slag Composition are Constant 


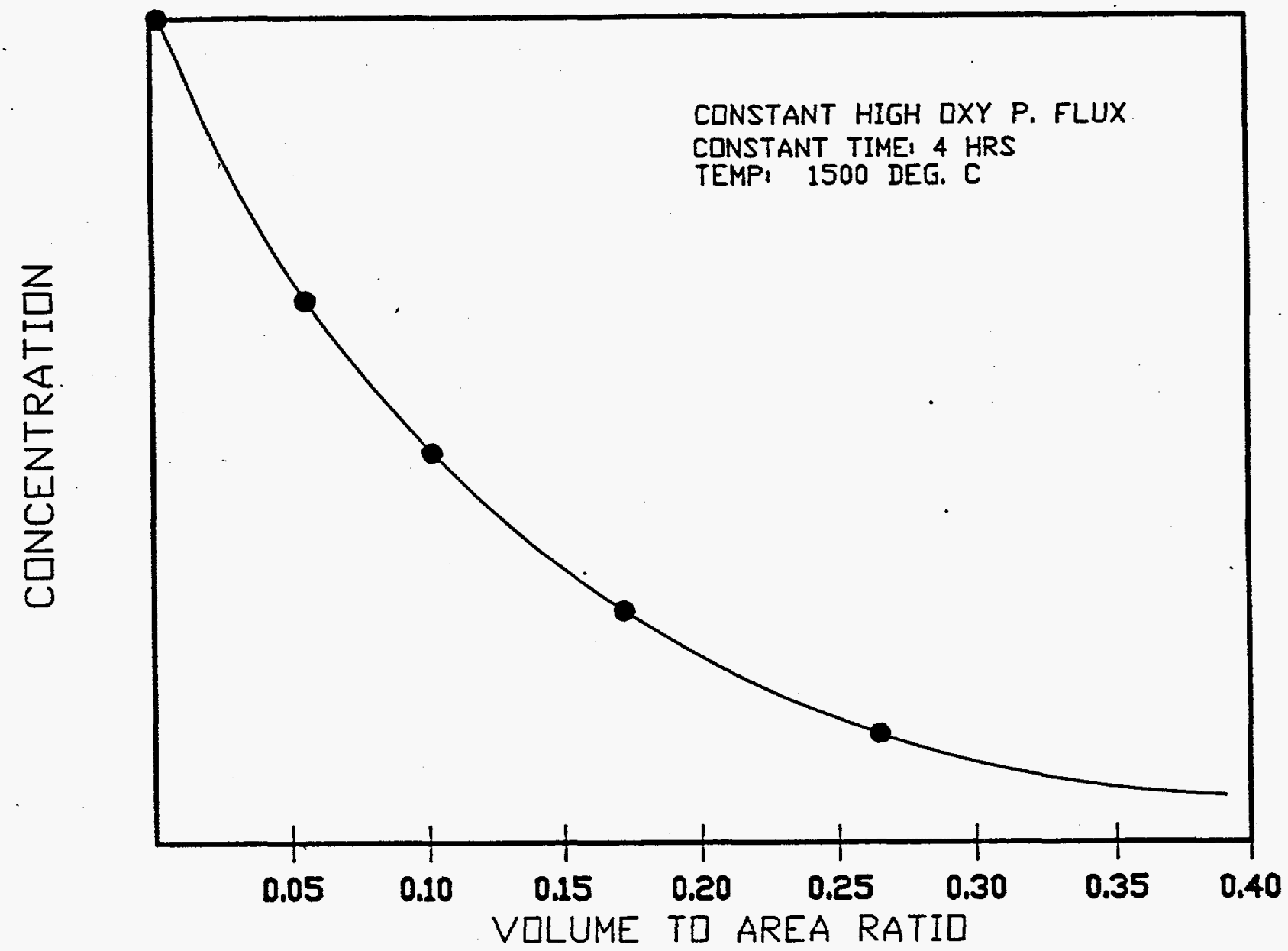

Figure 11. Anticipated Results from the Partitioning Experiments where Slag Composition, Temperature, and Time are Kept Constant and the Volume to SurfaceArea Ratio of the Slag / Metal Interface is Allowed to Change 


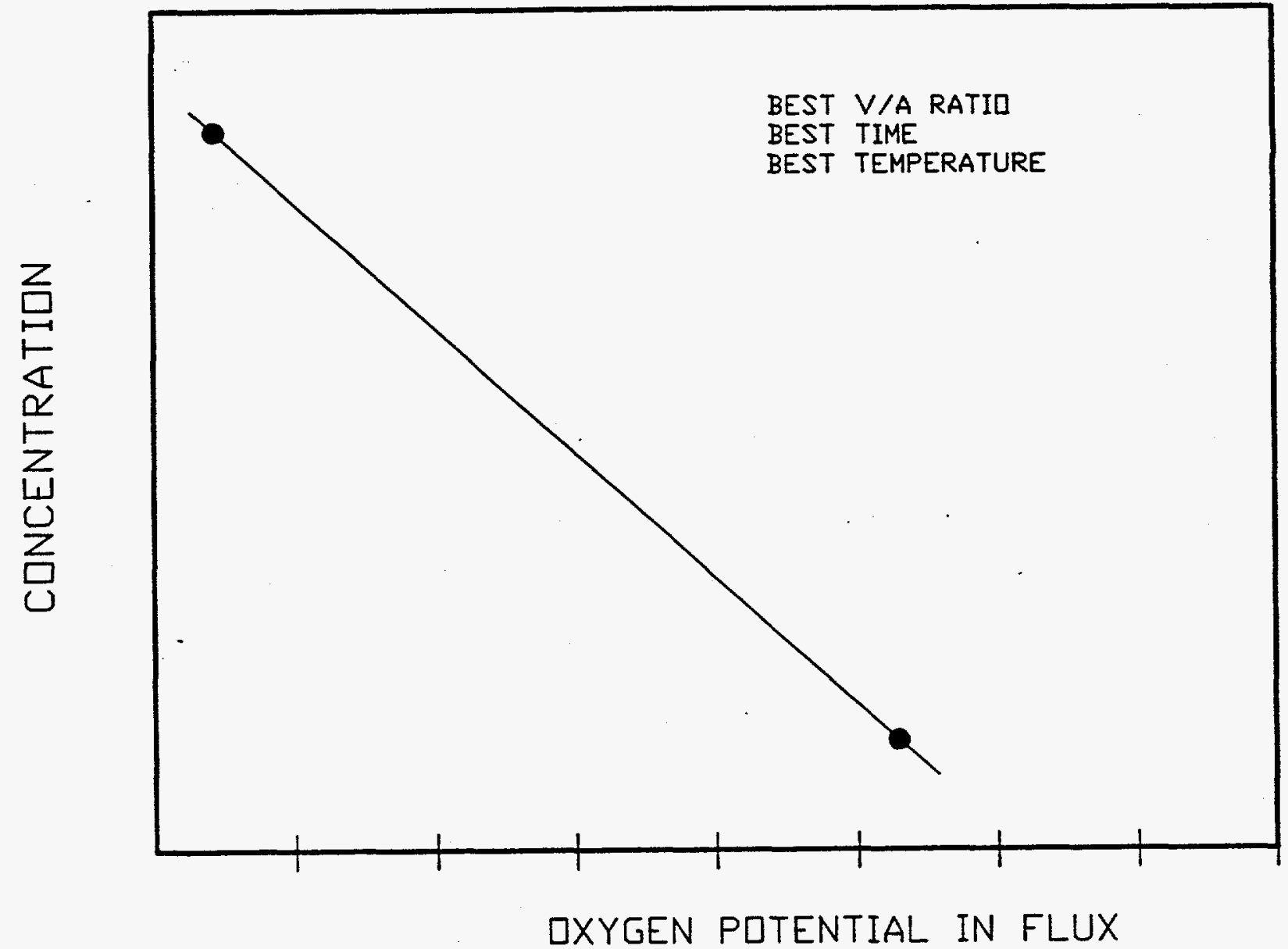

Figure 12. Anticipated Results from the Partitioning Experiments where Slag Composition is Changed, and Temperature, Time, and Volume to Surface Area Ratio of the Slag / Metal Interface are Kept Constant 